\title{
MicroRNA-18a upregulates autophagy and ataxia telangiectasia mutated gene expression in HCT116 colon cancer cells
}

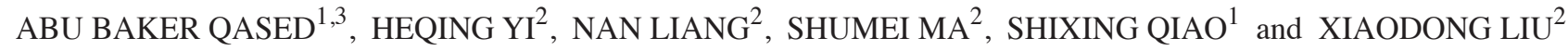 \\ ${ }^{1}$ Department of Surgery, The Second Hospital of Jilin University, Changchun, Jilin; ${ }^{2}$ Key Laboratory \\ of Radiobiology (Ministry of Health), School of Public Health, Jilin University, Changchun, Jilin, \\ P.R. China; ${ }^{3}$ Department of Surgery, College of Medicine, University of Mosul, Mosul, Iraq
}

Received August 19, 2012; Accepted November 22, 2012

DOI: $10.3892 / \mathrm{mmr} .2012 .1214$

\begin{abstract}
Autophagy is an evolutionarily conserved, multi-step lysosomal degradation process in which a cell degrades its own long-lived proteins and damaged organelles. Ataxia telangiectasia mutated (ATM) has recently been shown to upregulate the process of autophagy. Previous studies showed that certain microRNAs, including miR-18a, potentially regulate ATM in cancer cells. However, the mechanisms behind the modulation of ATM by miR-18a remain to be elucidated in colon cancer cells. In the present study, we explored the impact of miR-18a on the autophagy process and ATM expression in HCT116 colon cancer cells. To determine whether a preliminary link exists between autophagy and miR-18a, HCT116 cells were irradiated and quantitative (q) PCR was performed to measure miR-18a expression. HCT116 cells were transfected with an miR-18a mimic to study its impact on indicators of autophagy. Western blotting and luciferase assays were implemented to explore the impact of miR-18a on ATM gene expression in HCT116 cells. The results showed that miR-18a expression was strongly stimulated by radiation. Ectopic overexpression of miR-18a in HCT116 cell lines potently enhanced autophagy and ionizing radiation-induced autophagy. Moreover, miR-18a overexpression led to the upregulation of ATM expression and suppression of mTORC1 activity. Results of the present study pertaining to the role of miR-18a in regulating autophagy and ATM gene expression in colon cancer cells revealed a novel function for miR-18a in a critical cellular event and on a crucial gene with significant impacts in cancer development, progression, treatment and in other diseases.
\end{abstract}

Correspondence to: Professor Shixing Qiao, Department of Surgery, The Second Hospital of Jilin University, 218 Ziqiang Street, Changchun, Jilin 130041, P.R. China

E-mail: shixingqiaojdey@yahoo.com

Professor Xiaodong Liu, Key Laboratory of Radiobiology (Ministry of Health), School of Public Health, Jilin University, 1163 Xinmin street, Changchun, Jilin 130021, P.R. China

E-mail: liuxiaod@jlu.edu.cn

Key words: ataxia telangiectasia mutated, miR-18a, autophagy, ionizing radiation, colon cancer

\section{Introduction}

Autophagy is a cellular catabolic degradation process which functions to optimize the bioenergetic cellular microenvironment by degrading long-lived proteins and damaged organelles. Autophagy uses programmed machinery that recruits a plethora of autophagy-related genes (ATGs) which are required for autophagosome synthesis. Autophagosomes are double membrane structures that sequester the intended cytoplasmic portion, then bind to the lysosome to form autophagolysosomes, where the cargo is digested (1). Autophagy is involved in various physiological and pathophysiological processes, including aging, cancer and neurodegenerative diseases (2).

MicroRNAs are a short, non-coding, novel class of gene regulators. They are synthesized in the nucleus, exported to the cytoplasm and eventually bind to the 3'-untranslated region (3'-UTR) of their target mRNA to repress gene expression at the post-transcriptional level. Findings of previous studies demonstrated the role of microRNAs in various cellular events, including growth, apoptosis and carcinogenesis (3). MicroRNAs have recently been characterized as modulating the process of autophagy via the targeting of cardinal autophagy-regulating genes. miR-30a and miR-376b have been demonstrated to target and inhibit Beclin-1 activity, thereby blocking autophagy $(4,5)$. Additionally, miR-101 inhibits RAB5A, which acts in the early stages of autophagosome formation (6).

Ataxia telangiectasia mutated (ATM) is a Ser/Thr protein kinase and a member of the phosphoinositide 3-kinase (PI3K)-related protein kinase (PIKK) family. ATM functions to maintain genomic stability by orchestrating the actions of several downstream substrates involved in cell cycle arrest, apoptosis and DNA repair in response to DNA damage-inducing agents, particularly ionizing radiation (7). ATM has also been identified to upregulate the autophagic response of cells to genotoxic and oxidative stimuli (8). It has been reported that the expression of ATM is modulated by miR-421, miR-101, miR-100 and miR-18a in cancer cells (9-12). miR-18a, a member of the miR-17-92 cluster, has been shown to be significantly overexpressed in colon cancer tissues in comparison to normal colon mucosal cells (13). Modulation of ATM expression by miR-18a has not been exhibited in colon 
cancer cells. In the present study, we explored the impact of miR-18a on the process of autophagy and ATM expression in colon cancer cells, using the colon cancer cell line HCT116.

\section{Materials and methods}

Cell lines and radiation. HCT116 colon cancer cells were grown in DMEM supplemented with 10\% fetal bovine serum, $2 \mu \mathrm{M}$ glutamine, $100 \mathrm{IU} / \mathrm{ml}$ penicillin and $100 \mu \mathrm{g} / \mathrm{ml}$ streptomycin sulfate, in humidified conditions in an incubator containing $5 \% \mathrm{CO}_{2}$ and at $37^{\circ} \mathrm{C}$. For the $\mathrm{X}$-ray irradiation procedure a 180-kVp X-ray generator (Model XSZ-Z20/20, China) was utilized at a dose rate of $0.41 \mathrm{~Gy} / \mathrm{min}$. The study was approved by the ethics committee of Jilin University School of Public Health. Informed consent was obtained from patients.

RNA extraction. The total RNA of the cultured cells was extracted with TRIzol reagent (Invitrogen, Carlsbad, CA, USA) according to the manufacturer's instructions and stored at $-80^{\circ} \mathrm{C}$ prior to RT-PCR analysis.

Quantitative (q) RT-PCR for measuring miR-18a expression. For the SYBR-Green assay miRCURY LNA ${ }^{\mathrm{TM}}$ Universal RT microRNA PCR was utilized (Exiqon, Denmark). RNA $(\sim 20 \mathrm{ng})$ was converted to cDNA using the miRCURY LNA Universal RT microRNA PCR with the Poly-T primer (Exiqon). Following reverse transcription, the cDNA template was amplified using microRNA-specific and LNA primers. qRT-PCR was performed using the Stratagene MX3000p thermocycler according to the manufacturer's instructions. The U6 gene was used as a normalization control for all samples.

Plasmids. To construct a plasmid expressing miR-18a, primiR-18a was amplified using the genomic DNA from a healthy blood donor. PCR was performed using the rTaq enzyme (Takara, Dalian, China). The amplified fragment was first cloned into a PCR cloning vector (PMD-19T) and subsequently cloned into a lentiviral vector (pCDHCMV-MCS-EF1copGFP; System Biosciences, Mountain View, CA, USA) at the EcoRI and BamHI sites. Primer sequences used were: hsa-miR-18a (forward), GCCGAATTCGTGCAGGTAGT GATATGTGC; hsa-miR-18a (reverse), CGCGGATCCGA TTTGCACAACTACATTC. The 3'-UTR of ATM (Genbank accession no. NM_000051.3, region between 9557 and $13147 \mathrm{bp}$ ) carrying the putative miR-18a binding site was amplified by PCR from the human genomic DNA of the blood and cloned between the SacI and HindIII sites of the pMIRREPORT $^{\mathrm{TM}}$ luciferase vector (Ambion, Foster City, CA, USA).

The primers selected were the upstream ATM 3'-UTR primer 5'-GCCTCTAGACTCCTGTTCTGTTCAAGTAT-3' and the downstream ATM 3'-UTR primer 5'-GGCCCTCTA GAGCTTTTAGAATTATTTATTC-3'. PCR products cloned into the plasmid were verified by DNA sequencing to ensure that they were free of mutations and in the correct cloning direction.

Luciferase reporter assays. HCT116 cells at a density of $0.5 \times 10^{5} /$ well were cultured in 24 -well plates and each well was transfected with $20 \mathrm{ng}$ pMIR-ATM-3'-UTR, together with 5 ng pRL-SV40 vector (Promega, Madison, WI, USA), which contains the Renilla luciferase gene, and 50 or $100 \mathrm{nM}$ of the miR-18a mimics/negative control (NC; GenePharma, Shanghai, China) or PCDH-miR-18a/PCDH-control. Transfection was performed using Lipofectamine ${ }^{\mathrm{TM}} 2000$ (Invitrogen). At $48 \mathrm{~h}$ post-transfection, firefly and Renilla luciferase activities were examined using the Dual-Luciferase Reporter Assay (Promega).

Western blot analysis. Cells were harvested and lysed in a RIPA lysis buffer (150 mM sodium chloride, $1.0 \%$ NP-40 or Triton X-100, 0.1\% SDS, $50 \mathrm{mM}$ Tris, $\mathrm{pH} 8.0,20 \mathrm{mmol} / 1$ $\mathrm{Na}_{2} \mathrm{PO}_{4}, \mathrm{pH} 7.4$, aprotinin, $1 \mathrm{mmol} / 1$ phenymethysulfonyl fluoride, $10 \mathrm{mg} / \mathrm{ml}$ leupeptin, $100 \mathrm{mmol} / \mathrm{l} \mathrm{NaF}$ and $2 \mathrm{mmol} / \mathrm{l}$ $\mathrm{Na}_{3} \mathrm{VO}_{4}$ ). Total protein $(100 \mu \mathrm{g})$ was separated by SDS-PAGE, transferred to nitrocellulose membranes and analyzed by immunoblotting using chemiluminescence (Santa Cruz Biotechnology, Inc. Santa Cruz, CA, USA). The primary antibodies used were ATM (1:300) and LC3-I/II (1:300; Cell Signaling Technology Inc., Danvers, MA, USA), GAPDH (1:1000; Santa Cruz Biotechnology), P62/SQSTM1 (1:500) and P70S6K and p-P70S6K (Thr389; 1:500; Abcam, Cambridge, MA, USA). The intensity of the protein bands was quantified using Image $\mathbf{J}$ software and the control was set to 1 .

GFP-LC3 study. To generate the stable expression of GFP-LC3, the HCT116 cells were transfected with the purified recombinant plasmid, pQN-GFP-LC3, using Lipofectamin ${ }^{\mathrm{TM}} 2000$ according to the manufacturer's instructions. The HCT116 cells stably expressing GFP-LC3 were plated at a density of $1 \times 10^{5}$ in a 6 -well plate with glass coverslips in the bottom and exposed to the indicated transfections of microRNA, then irradiated. GFP-LC3 puncta were visualized under an inverted fluorescence (Olympus XSZ-D2) microscope equipped with $\mathrm{CCD}$ cameras. Images were captured for each sample at $48 \mathrm{~h}$ post-transfection, then analyzed manually.

Statistical analysis. The Student's t-test was used to determine statistical significance. $\mathrm{P}<0.01$ and $\mathrm{P}<0.05$ were considered to indicate statistically significant differences.

\section{Results}

Endogenous miR-18a expression upregulation by ionizing radiation. To determine whether a preliminary link exists between autophagy and miR-18a expression we measured the level of endogenous miR-18a under basal growth conditions and following exposure to ionizing radiation (IR) in HCT116 colon cancer cells. Quantitative RT-PCR was performed using the HCT116 cells collected following exposure to $4 \mathrm{~Gy}$ of radiation. The expression level of miR-18a markedly increased by $>200$-fold $1 \mathrm{~h}$ after irradiation (Fig. 1).

Ectopic miR-18a overexpression promotes autophagy. During autophagy, the mammalian ATG8-homolog (LC3-I) is processed and recruited to the autophagosomes, where the lipidated LC3-II is generated (14). Therefore, HCT116 cells stably expressing GFP-LC3 were transfected with a miR-18a mimic, a negative control or left without any transfection as mock cells. At $48 \mathrm{~h}$ post-transfection, analysis by fluorescence microscopy for the presence of GFP-LC3 puncta was performed (Fig. 2A). miR-18a expression increased the percentage of 


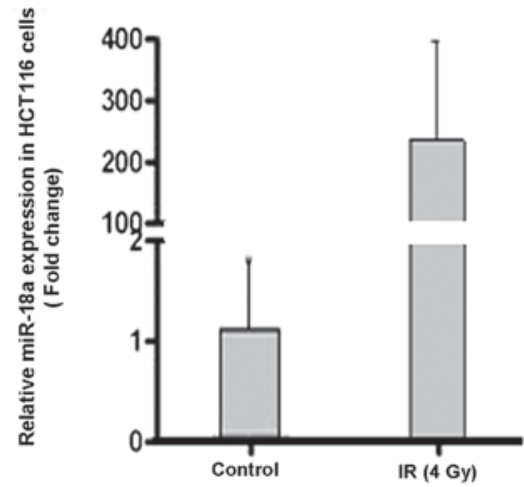

Figure 1. Endogenous miR-18a expression increased following exposure of HCT116 cells to IR. HCT116 cells were exposed to 4 Gy of ionizing radiation, and then qRT-PCR was performed to measure the relative expression level of miR-18a. This expression level was compared with the control cells to show the fold changes $(n=3)$. qRT-PCR, quantitative RT-PCR; IR, ionizing radiation.

puncta-positive cells to a significant extent as compared with the NC-transfected and mock cells (Fig. 2B). Notably, the percentage of puncta-positive cells was further enhanced when miR-18a was combined with IR, relative to cells treated with IR alone (Fig. 2C and D). Similarly, miR-18a overexpression enhanced the LC3-II protein expression level in non-irradiated and irradiated HCT116 cells (Fig. 2E and F). P62/SQSTM1 is a poly-ubiquitin binding protein that was identified as being able to bind directly to ATG8/LC3 and localize to autophagosomes to ultimately be degraded during autophagy. Therefore, the level of P62 reflects the autophagic turnover (15). As shown in Fig. 2G and H, in the HCT116 cells transfected with miR-18a the expression level of the P62/SQSTM1 protein was markedly reduced in non-irradiated and irradiated cells as compared with the NC-transfected cells. Consequently, such findings demonstrated that miR-18a enhanced the autophagic flux. Taken together, these results indicate that miR-18a upregulates the process of autophagy in the HCT116 cell line and potentiates the autophagic response of HCT116 cells to radiation.

Ectopic miR-18a overexpression upregulates ATM gene expression. First, we explored the effect of miR-18a on endogenous ATM protein levels in HCT116 cells by western blotting. Using a miR-18a mimic, we identified that an ectopic increase in miR-18a led to $>50 \%$ increase in ATM protein levels in nonirradiated and irradiated HCT116 cells relative to the negative control (NC) transfected cells (Fig. 3A and B). Opposite trends were identified in the cells transfected with the miR-18a inhibitor (Fig. 3C and D). According to the databases from three popular microRNA target prediction programs (Targetscan, miranda and miRBase), the ATM mRNA 3'-UTR contains a sequence motif (position 3481-3488) which is complementary to the miR-18a seed sequence and may be a putative binding site for miR-18a (Fig. 3E). Therefore, we constructed a luciferase reporter plasmid (pMIR-ATM 3'-UTR) with the ATM
A
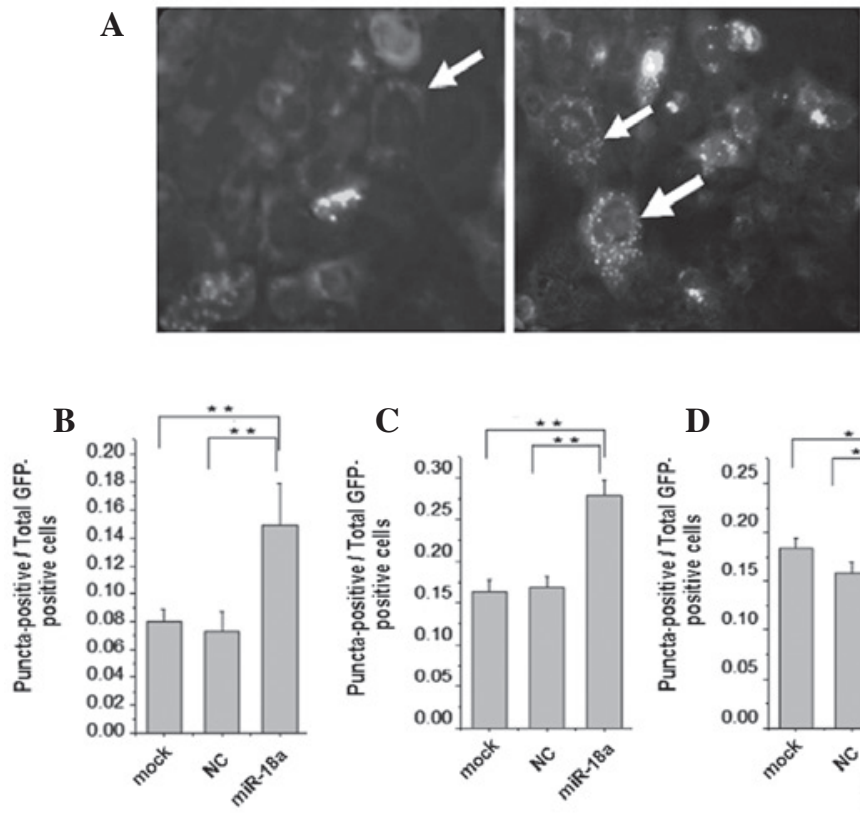

HCT116 cells

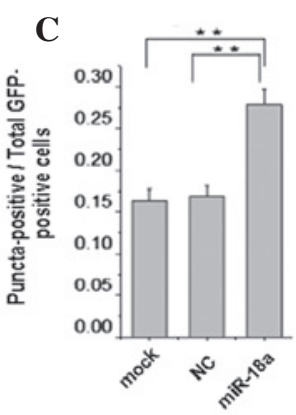

HCT116 cells+ IR (4 Gy)

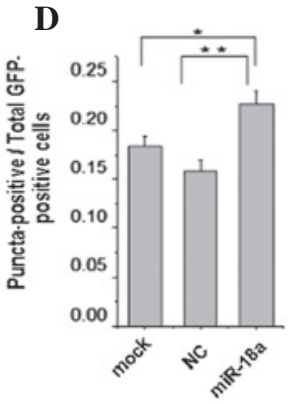

HCT116 cells + IR (8Gy)
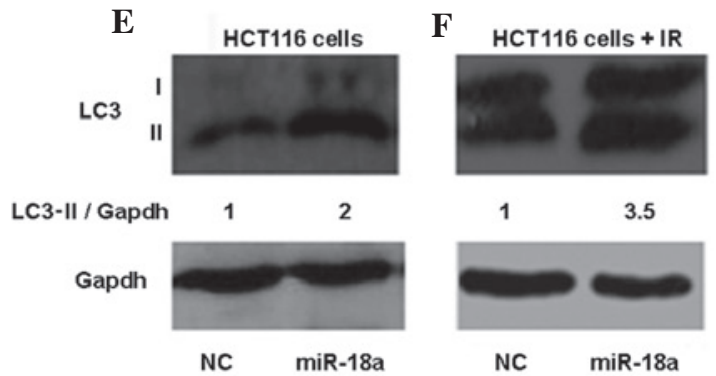

1

3.5

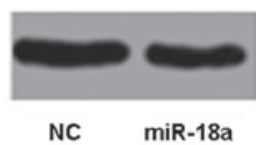

G

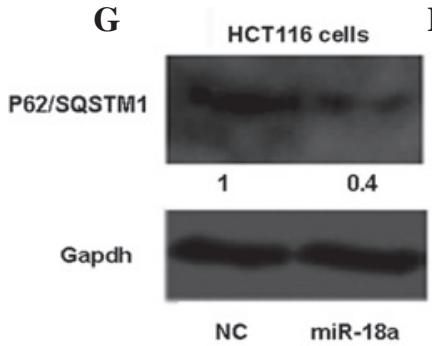

H

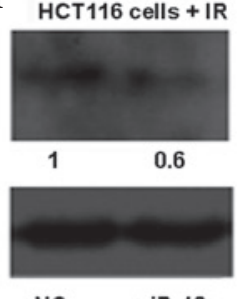

NC

$\operatorname{miR}-18 \mathrm{a}$

Figure 2. Ectopic miR-18a overexpression upregulates autophagy in HCT116 cells. (A) Representative images of HCT116 cells stably expressing GFP-LC3; the right panel with the white arrows indicating a typical puncta-positive cell shows induction of autophagy, while the left panel with a white arrow indicating a GFPLC3-positive cell shows that autophagy was not induced. (B-D) Stably expressing GFP-LC3 HCT116 cells were transfected with the negative control (NC) or miR-18a mimic $(100 \mathrm{nM})$ or left without transfection as mock cells, then treated accordingly with indicated doses of radiation. The graphs show the total number of puncta-positive cells divided by the total GFP-positive cells; ${ }^{*} \mathrm{P}<0.05,{ }^{* *} \mathrm{P}<0.01$ compared to mock or NC. (E) miR-18a overexpression increases LC3-II protein expression. HCT116 cells were transfected with NC or miR-18a mimic (100 nM) then the cell lysate was applied to western blot analysis $48 \mathrm{~h}$ post-transfection. GAPDH was used as a loading control, $n=3$. (F) HCT116 cells were transfected with NC or miR-18a mimic (100 nM) then treated with 4 Gy of IR. Western blot analysis was conducted $48 \mathrm{~h}$ post-transfection (20 h post-radiation) to measure LC3-II protein expression, $\mathrm{n}=2$. (G) miR-18a overexpression led to a decreased expression of the P62/SQSTM1 protein. HCT116 cells were transfected with NC or miR-18a mimic (100 nM), then the cell lysate was exposed to western blot analysis $48 \mathrm{~h}$ post-transfection, $\mathrm{n}=2$. (H) HCT116 cells were transfected with NC or miR-18a mimic (100 nM), then treated with 4 Gy of radiation. The cell lysate was subjected to western blot analysis $48 \mathrm{~h}$ post-transfection $(20 \mathrm{~h}$ post-IR) to measure the P62/SQSTM1 expression level, $\mathrm{n}=2$. IR, ionizing radiation. 
A

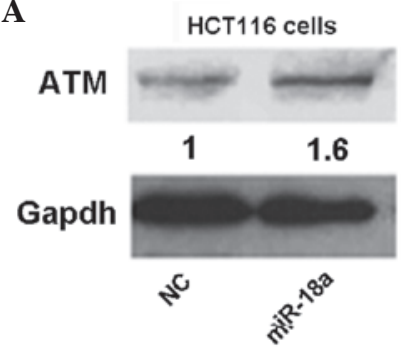

$\mathbf{E}$

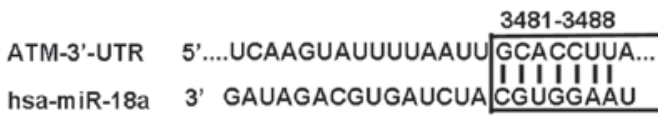

G
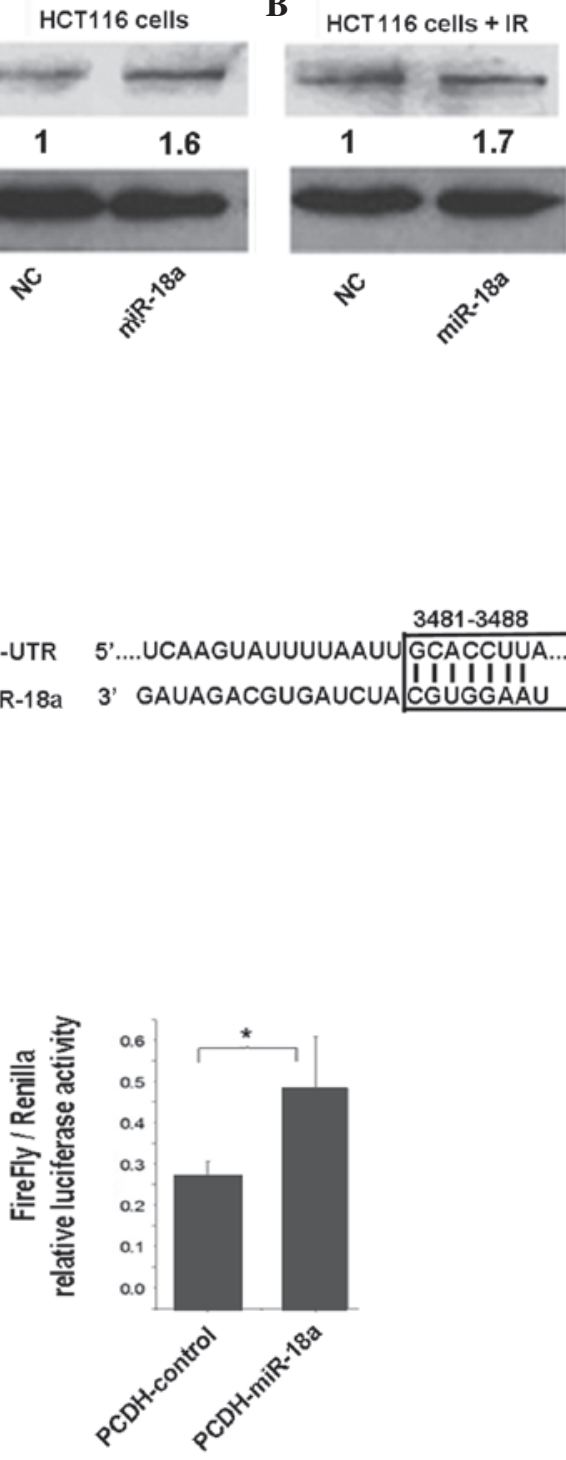

C

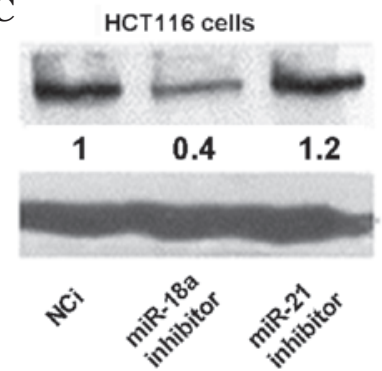

D

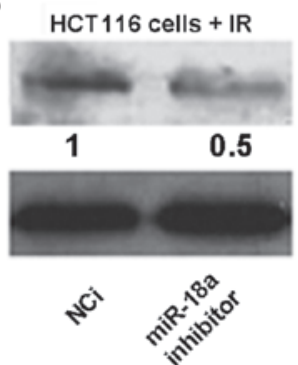

$\mathbf{F}$

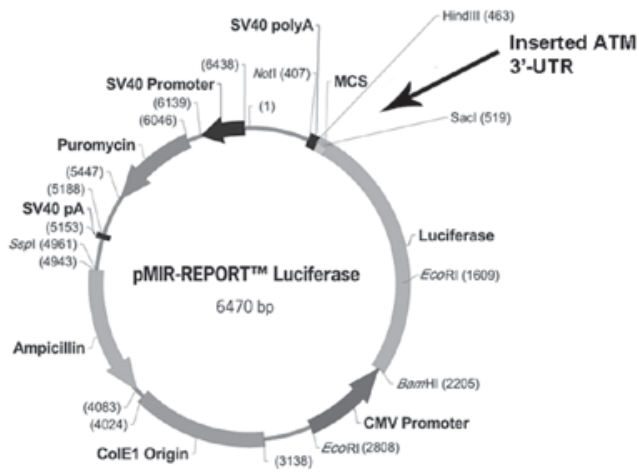

$\mathbf{H}$

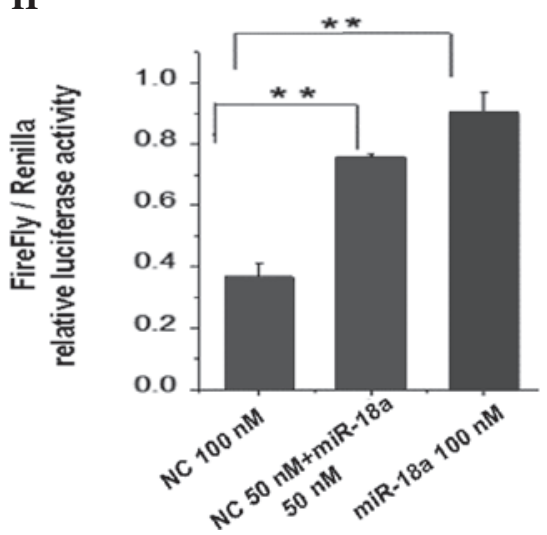

Figure 3. Ectopic miR-18a overexpression upregulates the ATM gene expression level in HCT116 cells. (A) HCT116 cells were harvested $48 \mathrm{~h}$ after transfection with the negative control (NC) or miR-18a mimic $(100 \mathrm{nM})$ and the cell lysate was applied to a western blot, $\mathrm{n}=3$. (B) HCT116 cells were transfected with the NC or miR-18a mimic (100 nM), irradiated with $4 \mathrm{~Gy}$, and the cell lysate was applied to a western blot $48 \mathrm{~h}$ post-transfection (20 h post-radiation), $\mathrm{n}=2$. (C) Suppression of ATM expression in HCT116 cells by miR-18a inhibitor. HCT116 cells were harvested $48 \mathrm{~h}$ after transfection with the negative control inhibitor $(\mathrm{NCi})$ or miR-18a inhibitor $(100 \mathrm{nM})$ and the cell lysate was applied to a western blot. miR-21 inhibitor was used as non-sense control, $\mathrm{n}=2$. (D) HCT116 cells were transfected with NCi or miR-18a inhibitor (100 nM) then irradiated with $4 \mathrm{~Gy}$, and cell lysate was applied to a western blot $48 \mathrm{~h}$ post-transfection ( $20 \mathrm{~h}$ post-radiation), $\mathrm{n}=2$. (E) The nucleotide segment within the box shows the seed sequence of miR-18a with its corresponding binding site in the 3'-UTR of ATM. (F) The ATM 3'-UTR segment carrying the putative miR-18a binding site was cloned between the SacI and HindIII sites of the pMIR-REPORT TM luciferase vector. (G and H) Luciferase reporter activity was assayed $48 \mathrm{~h}$ post-transfection of the HCT116 cell line with vector expressed PCDH-miR-18a or synthetic miR-18a mimic $(100 \mathrm{nM})$ and the pMIR-luciferase reporter with the partial 3'-UTR of ATM. Relative luciferase activity was normalized to Renilla luciferase and compared with the PCDH-empty vector (PCDH-control) $(n=2)$ or NC-transfected cells $(n=6) .{ }^{*} \mathrm{P}<0.05,{ }^{* *} \mathrm{P}<0.01$. ATM, ataxia telangiectasia mutated.

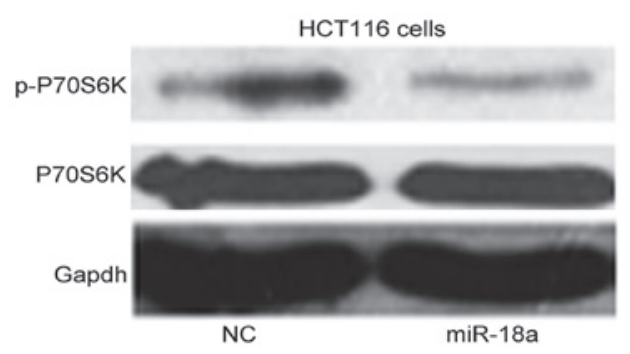

Figure 4. Ectopic miR-18a overexpression suppresses mTORC1 activity. HCT116 cells were transfected with miR-18a mimic $(100 \mathrm{nM})$ or negative control (NC). Western blot analysis was then conducted $48 \mathrm{~h}$ post-transfection to measure total P70S6K and phosphorylated P70S6K (p-P70S6K), n=2.
3'-UTR, which contains a putative binding site to miR-18a, and cloned it to a firefly luciferase reporter (Fig. 3F). PCDH vector-expressed miR-18a (PCDH-miR-18a) and synthetic miR-18a (miR-18a mimic) were then used to evaluate the effects of the microRNA on the reporter gene expression. At $48 \mathrm{~h}$ post-transfection, the luciferase activity was assayed and normalized to Renilla. In HCT116 cells, PCDH-miR-18a and the miR-18a mimic significantly increased the expression of the reporter gene with the ATM 3'-UTR tag (Fig. 3G and H). These results demonstrate for the first time that miR-18a is able to enhance ATM gene expression in the HCT116 colon cancer cell line, most likely by targeting the ATM 3'-UTR. 
miR-18a overexpression inhibits mTORC1 activity. ATM has been demonstrated to upregulate autophagy via inhibition of mTORC1 (8). We hypothesized that miR-18a positively regulated ATM, inhibiting mTORC1 and inducing autophagy. To examine whether miR-18a regulated mTORC1, we measured P70S6K phosphorylation at Thr389 as a typical readout of mTORC1 activity (16). As shown in Fig. 4, miR-18a overexpression resulted in hypophosphorylation of P70S6K (which indicated mTORC1 activity inhibition) compared with NC-transfected cells.

\section{Discussion}

Our knowledge concerning the molecular control of autophagy has greatly increased during the last decade, yet mechanisms controlling autophagic activity are not fully understood. Abnormalities of autophagy play a role in major health problems, including cancer and neurodegenerative diseases. The exact role of autophagy in carcinogenesis remains elusive. Autophagy is able to behave as a tumor suppressor or oncogene depending on the cell context (17). A similar paradox is exhibited during tumor therapy. Autophagy has been shown to support cancer cell survival, as has been observed in breast cancer cells (18). However, in other contexts, as in HCT116 colon cancer cells, autophagy has been shown to contribute to cell death $(19,20)$. Therefore, specific modulators of autophagy suitable for in vivo use are required (21). MicroRNAs have emerged recently as a novel class of endogenous gene regulators, and have been implicated in various pathological and physiological processes. Previously, a series of studies had been working to demonstrate the role of microRNAs in the regulation of autophagy. miR$376 \mathrm{~b}$ overexpression was shown to attenuate starvation- and rapamycin-induced autophagy in $\mathrm{MCF}-7$ and Huh-7 cancer cells by suppressing the autophagy proteins ATG4C and BECN1 as target genes (5). Other microRNAs, including miR-30a (via suppression of Beclin-1), miR-101 (via suppression of STMN1, RAB5A and ATG4D) and miR-204 (via blocking the activation of LC3-II activity), have all been shown to be potent suppressors of autophagy in cancer cells $(4,6,22)$.

In the present study we showed that miR-18a overexpression resulted in a significant promotion of autophagy in HCT116 colon cancer cells, as evidenced by increased GFP-LC3 puncta formation, increased LC3-II protein expression and a decreased P62/SQSTM1 protein level. Moreover, miR-18a promoted radiation-induced autophagy (Fig. 2). Additionally, the role of miR-18a in modulating autophagy is supported by our analysis of endogenous microRNA expression, which demonstrated a marked upregulation of miR-18a expression in HCT116 cells following exposure to radiation (Fig. 1). ATM encodes a 370-kDa protein with a carboxyl-terminal sequence homologous to the catalytic domain of phosphatidylinositol 3-kinases. Its classical function is to maintain genomic stability following the exposure of cells to agents that induce DNA damage (double strand breaks), particularly ionizing radiation, by phosphorylation of several downstream substrates involved in cell cycle arrest, apoptosis and DNA repair (23). ATM has been identified to upregulate the autophagic response of cells to genotoxic and oxidative stimuli. ATM stimulates downstream signaling through the LBK/AMPK/TSC2 pathway, which in turn results in $\mathrm{mTORC} 1$ repression and autophagy induction $(8,24,25)$. Therefore, ATM may serve as a direct link between DNA damage and autophagy.

The 3'-UTR segment of ATM mRNA contains only a single binding site for the seed sequence of miR-18a. We showed that miR-18a upregulated ATM gene expression in non-irradiated and 4 Gy-irradiated HCT116 cells, as evidenced by western blotting. Conversely, inhibition of miR-18a led to the suppression of ATM protein expression. To explore the mechanisms we constructed plasmids containing the binding site for miR-18a and conducted a dual-luciferase reporter assay. Following co-transfection, luciferase activity with the ATM 3'-UTR construct increased significantly in the miR-18a mimic and vector expressed miR-18a (PCDH-miR-18a)-transfected cells (Fig. 3). These results suggest that ATM expression may be elevated by miR-18a through targeting of the 3'-UTR of ATM mRNA. However, the theory that overexpression of miR-18a downregulates ATM expression in breast cancer cells remains controversial (12). Similar controversial findings have been exhibited by miR-21, which has been to shown to upregulate $\mathrm{Bcl}-2$ gene expression in pancreatic cancer cells, but also to lead to downregulation of Bcl-2 in breast cancer cells $(26,27)$. Previous studies have revealed that certain microRNAs are able to directly upregulate the expression of their target genes by binding to the 3 '-UTR $(28,29)$. Collectively such findings suggest that the impact of miR-18a on ATM gene expression may be cell-type specific. Results from the present study demonstrate for the first time that miR-18a is able to upregulate ATM target gene expression in HCT116 cells and provides a novel clue for exploring the exact targets and mechanisms of miR-18a in colon cancer cells. Further comprehensive investigations are consequently required. This provides a new challenge to further understand the mechanisms of microRNAs. As it is well known that microRNAs target several genes simultaneously, we hypothesize that the miR-18a induces autophagy, at least partially, through targeting the ATM gene. This hypothesis is supported partially by our finding that miR-18a overexpression suppressed mTORC1 activity (Fig. 4).

Colorectal cancer (CRC) is the second most common cause of cancer mortality in the western world. CRC develops from an accumulation of multiple genetic and epigenetic alterations that contribute to its diverse phenotypes. Genomic instability, which occurs in $\sim 5 \%$ of colorectal adenomas, constitutes a major step in the progression to cancer $(30,31)$. ATM, the guardian of genomic integrity, has been shown to play a major tumor suppressive role in colon cancer by inhibiting the progression of pre-neoplastic lesions into neoplasia $(32,33)$. miR-18a belongs to the miR-17-92 cluster, located at 13q, which encodes six microRNAs processed from a common precursor transcript. The miR-17-92 cluster has been shown to be involved in the pathogenesis of human cancers, including diffuse large B cell lymphoma (34) and small cell lung cancer (35). However, the pathophysiological roles and targets of members of this cluster, particularly miR-18a, are largely unknown in colon cancer (36). The results of the present study suggest that miR-18a has the potential to modulate autophagy through regulation of the expression of the known autophagy activator ATM, thus providing evidence for a new role of miR-18a in a cellular process with a significance that has increasingly been recognized in cancer biology. An improved understanding of the microRNA-modulated autophagic signaling networks is 
likely to be crucial to the current and future cancer therapeutic strategies (37). The ability of miR-18a to upregulate ATM gene expression, which has potent antitumor features, renders miR-18a a good candidate for future studies in the field of microRNA-based epigenetic colon cancer therapy.

\section{Acknowledgements}

The authors would like to thank Dr Yin-Yuan Mo (Department of Medical Microbiology, Immunology and Cell Biology, Southern Illinois University School of Medicine, Springfield, IL, USA) for providing them with a (pCDHCMVMCS-EF1-copGFP) lenti-vector. They are also grateful to Mr. Song Zhiheng for his technical assistance. This study was sponsored by an NSFC grant $(30770649,30970682)$ and the Research Fund for the Doctoral Program of Higher Education of China (20100061110070).

\section{References}

1. Mizushima N: Autophagy: process and function. Genes Dev 21: 2861-2873, 2007.

2. Levine B and Kroemer G: Autophagy in the pathogenesis of disease. Cell 132: 27-42, 2008

3. He L and Hannon GJ: MicroRNAs: small RNAs with a big role in gene regulation. Nat Rev Genet 5: 522-531, 2004.

4. Zhu H, Wu H, Liu X, Li B, Chen Y, Ren X, Liu CG and Yang JM: Regulation of autophagy by a beclin 1-targeted microRNA, miR-30a, in cancer cells. Autophagy 5: 816-823, 2009.

5. Korkmaz G, le Sage C, Tekirdag KA, Agami R and Gozuacik D: miR-376b controls starvation and mTOR inhibition-related autophagy by targeting ATG4C and BECN1. Autophagy 8: $165-176,2012$

6. Frankel LB, Wen J, Lees M, Høyer-Hansen M, Farkas T, Krogh A, Jäättelä M and Lund AH: microRNA-101 is a potent inhibitor of autophagy. EMBO J 30: 4628-4641, 2011.

7. Derheimer FA and Kastan MB: Multiple roles of ATM in monitoring and maintaining DNA integrity. FEBS Lett 584: 3675-3681, 2010.

8. Alexander A, Cai SL, Kim J, Nanez A, Sahin M, MacLean KH, Inoki K, Guan KL, Shen J, Person MD, Kusewitt D, Mills GB, Kastan MB and Walker CL: ATM signals to TSC2 in the cytoplasm to regulate mTORC1 in response to ROS. Proc Natl Acad Sci USA 107: 4153-4158, 2010.

9. Hu H, Du L, Nagabayashi G, Seeger RC and Gatti RA: ATM is down-regulated by N-Myc-regulated microRNA-421. Proc Natl Acad Sci USA 107: 1506-1511, 2010.

10. Ng WL, Yan D, Zhang X, Mo YY and Wang Y: Over-expression of miR-100 is responsible for the low-expression of ATM in the human glioma cell line: M059J. DNA Repair (Amst) 9: 1170-1175, 2010.

11. Yan D, Ng WL, Zhang X, Wang P, Zhang Z, Mo YY, Mao H, Hao C, Olson JJ, Curran WJ and Wang Y: Targeting DNA-PKcs and ATM with miR-101 sensitizes tumors to radiation. PLoS One 5: e11397, 2010.

12. Song L, Lin C, Wu Z, Gong H, Zeng Y, Wu J, Li M and Li J: miR-18a impairs DNA damage response through downregulation of ataxia telangiectasia mutated (ATM) kinase. PLoS One 6: e25454, 2011.

13. Tsuchida A, Ohno S, Wu W, Borjigin N, Fujita K, Aoki T, Ueda S, Takanashi M and Kuroda M: Cancer miR-92 is a key oncogenic component of the miR-17-92 cluster in colon cancer. Cancer Sci 102: 2264-2271, 2011

14. Klionsky DJ, Abeliovich H, Agostinis P, et al: Guidelines for the use and interpretation of assays for monitoring autophagy in higher eukaryotes. Autophagy 4: 151-175, 2008.

15. Pankiv S, Clausen TH, Lamark T, Brech A, Bruun JA, Outzen H, Øvervatn A, Bjørkøy G and Johansen T: p62/SQSTM1 binds directly to Atg8/LC3 to facilitate degradation of ubiquitinated protein aggregates by autophagy. J Biol Chem 282: 24131-24145, 2007.
16. Corradetti MN and Guan KL: Upstream of the mammalian target of rapamycin: do all roads pass through mTOR? Oncogene 25: 6347-6360, 2006.

17. Wu WK, Coffelt SB, Cho CH, Wang XJ, Lee CW, Chan FK, $\mathrm{Yu} \mathrm{J}$ and Sung JJ: The autophagic paradox in cancer therapy. Oncogene 31: 939-953, 2012.

18. Chaachouay H, Ohneseit P, Toulany M, Kehlbach R, Multhoff G and Rodemann HP: Autophagy contributes to resistance of tumor cells to ionizing radiation. Radiother Oncol 99: 287-292, 2011.

19. Ko H, Kim YJ, Amor EC, Lee JW, Kim HC, Kim HJ and Yang HO: Induction of autophagy by dimethyl cardamonin is associated with proliferative arrest in human colorectal carcinoma HCT116 and LOVO cells. J Cell Biochem 112: 2471-2479, 2011.

20. Yuk JM, Shin DM, Song KS, Lim K, Kim KH, Lee SH, Kim JM, Lee JS, Paik TH, Kim JS and Jo EK: Bacillus calmette-guerin cell wall cytoskeleton enhances colon cancer radiosensitivity through autophagy. Autophagy 6: 46-60, 2010.

21. Høyer-Hansen M and Jäättelä M: Autophagy: an emerging target for cancer therapy. Autophagy 4: 574-580, 2008.

22. Xiao J, Zhu X, He B, Zhang Y, Kang B, Wang Z and Ni X: MiR-204 regulate cardiomyocyte autophagy induced by hypoxiareoxygenation through LC3-II. Int J Cardiol 148: 110-112, 2011.

23. Shiloh Y: ATM and related protein kinases: safeguarding genome integrity. Nat Rev Cancer 3: 155-168, 2003.

24. Alexander A and Walker CL: The role of LKB1 and AMPK in cellular responses to stress and damage. FEBS Lett 585: 952-957, 2011.

25. Zhou WJ, Deng R, Zhang XY, Feng GK, Gu LQ and Zhu XF: G-quadruplex ligand SYUIQ-5 induces autophagy by telomere damage and TRF2 delocalization in cancer cells. Mol Cancer Ther 8: 3203-3213, 2009.

26. Dong J, Zhao YP, Zhou L, Zhang TP and Chen G: Bcl-2 upregulation induced by miR-21 via a direct interaction is associated with apoptosis and chemoresistance in MIA PaCa-2 pancreatic cancer cells. Arch Med Res 42: 8-14, 2011.

27. Wickramasinghe NS, Manavalan TT, Dougherty SM, Riggs KA, $\mathrm{Li} \mathrm{Y}$ and Klinge CM: Estradiol downregulates miR-21 expression and increases miR-21 target gene expression in MCF-7 breast cancer cells. Nucleic Acids Res 37: 2584-2595, 2009.

28. Vasudevan S, Tong Y and Steitz JA: Switching from repression to activation: microRNAs can up-regulate translation. Science 318: 1931-1934, 2007.

29. Vasudevan S, Tong Y and Steitz JA: Cell cycle control of microRNA-mediated translation regulation. Cell Cycle 7: 1545-1549, 2008.

30. Jemal A, Siegel R, Ward E, Murray T, Xu J and Thun MJ: Cancer statistics. CA Cancer J Clin 57: 43-66, 2007.

31. Hermsen M, Postma C, Baak J, Weiss M, Rapallo A, Sciutto A, Roemen G, Arends JW, Williams R, Giaretti W, De Goeij A and Meijer G: Colorectal adenoma to carcinoma progression follows multiple pathways of chromosomal instability. Gastroenterology 123: 1109-1119, 2002.

32. Bartkova J, Horejsí Z, Koed K, Krämer A, Tort F, Zieger K, Guldberg P, Sehested M, Nesland JM, Lukas C, Ørntoft T, Lukas $\mathrm{J}$ and Bartek J: DNA damage response as a candidate anti-cancer barrier in early human tumorigenesis. Nature 434: 864-870, 2005.

33. Bartek J, Bartkova J and Lukas J: DNA damage signalling guards against activated oncogenes and tumour progression. Oncogene 26: 7773-7779, 2007.

34. Ota A, Tagawa H, Karnan S, Tsuzuki S, Karpas A, Kira S, Yoshida $\mathrm{Y}$ and Seto M: Identification and characterization of a novel gene, C13orf25, as a target for 13q31-q32 amplification in malignant lymphoma. Cancer Res 64: 3087-3095, 2004.

35. Hayashita Y, Osada H, Tatematsu Y, Yamada H, Yanagisawa K, Tomida S, Yatabe Y, Kawahara K, Sekido Y and Takahashi T: A polycistronic microRNA cluster, miR-17-92, is overexpressed in human lung cancers and enhances cell proliferation. Cancer Res 65: 9628-9632, 2005.

36. van Haaften G and Agami R: Tumorigenicity of the miR-17-92 cluster distilled. Genes Dev 24: 1-4, 2010.

37. Fu LL, Wen X, Bao JK and Liu B: MicroRNA-modulated autophagic signaling networks in cancer. Int J Biochem Cell Biol 44: 733-736, 2012. 